\title{
Boundedness and Stability of Impulsively Perturbed Systems in a Banach Space
}

\author{
L. Berezansky ${ }^{1}$ and E. Braverman ${ }^{2}$
}

October 26, 2018

\begin{abstract}
Running head: Stability of impulsively perturbed systems Corresponding author: E. Braverman

Correspondent's phone: 972-429-4020

Correspondent's e-mail: maelena@tx.technion.ac.il
\end{abstract}

\begin{abstract}
We consider the influence of impulsive perturbations of a linear impulsive equation in a Banach space on the existence of bounded solutions and the exponential stability of the equation.
\end{abstract}

\section{Introduction}

The theory of impulsive differential equations goes back to the work (Millman and Myshkis, 1960). These equations describe processes changing their state abruptly at certain moments. This means that the duration of the perturbation is negligible comparing with the time of the process. Perturbations of this kind occur in control problems (Bressan and Rampazzo, 1991). Recently the development of the theory of impulsive differential equations in

\footnotetext{
${ }^{1}$ Ben-Gurion University of the Negev, Department of Mathematics and Computer Science, Beer-Sheva 84105, Israel

${ }^{2}$ Technion-Israel Institute of Technology, Department of Mathematics, Haifa 32000, Israel
} 
abstract spaces has begun (Bainov et al., 1988 both, 1989, 1993, Zabreiko et al., 1988).

We consider the problem

$$
\begin{gathered}
\dot{x}+A(t) x(t)=f(t), t \in[0, \infty), \\
x\left(\tau_{i}+0\right)=B_{i} x\left(\tau_{i}-0\right),
\end{gathered}
$$

where $x(t)$ is an element of a Banach space $Y, B_{i}: Y \rightarrow Y$ are linear bounded operators. Our objective is to derive conditions such that the solution $x$ is bounded on the half-line $[0, \infty)$ for any bounded right-hand side $f$ :

$$
\sup _{t \geq 0}\|f(t)\|<\infty
$$

where $\|\cdot\|$ is the norm in $Y$. In the control theory this problem is treated as follows. Is the output $x$ bounded for any bounded input $f$ ?

Naturally the above problem is connected with the exponential stability of a differential equation. The work (Anokhin et al.) deals with results of this kind for impulsive delay differential equations. The results for differential equations without impulses in a Banach space can be found in (Daleckii and Krein, 1974). For impulsive differential equations in a Banach space a connection of boundedness and stability is obtained in (Zabreiko et al., 1988).

Our approach is different. We consider the homogeneous equation

$$
\dot{x}(t)+A(t) x(t)=0, t \in[0, \infty),
$$

with non-homogeneous impulsive conditions

$$
x\left(\tau_{i}+0\right)=B_{i} x\left(\tau_{i}-0\right)+\alpha_{i}, i=1,2, \ldots,
$$

where

$$
\sup _{i}\left\|\alpha_{i}\right\|<\infty
$$

Suppose any solution of (4),(5), with (6), is bounded on the half-line. We prove that under natural constraints each solution of (1),(2), with the right-hand side satisfying (3), is also bounded on the half-line. 
It is to be emphasized that unlike (Zabreiko et al., 1988) we do not need to check whether the solution is bounded for any bounded right-hand side. Moreover, in the scalar case $Y=\mathbf{R}$ we can choose certain $\alpha_{i}$ (precisely, $\alpha_{i}=\operatorname{sign}\left\{\prod_{j=1}^{i} B_{j}\right\}$, where sign $\left.u=u /|u|\right)$. Then by checking the boundedness of $x$ in the only case we obtain that the solution of (1),(5) is bounded for any $f, \alpha$ satisfying (3),(6). The result can be applied to differential equations without impulses and it is new for them.

The paper is organized as follows. First we obtain that if a solution of each problem (4),(5), with (6), is bounded on the half-line, then the evolution operator has an exponential estimate. The section 3 deals with the main result described above. In the section 4 the connection between impulsive equations and equations without impulses is considered.

\section{Exponential estimates}

Let $0=\tau_{0}<\tau_{1}<\ldots$ be fixed points, $\lim _{i \rightarrow \infty} \tau_{i}=\infty, Y$ be a Banach space with a norm $\|\cdot\|$, by $\|\cdot\|$ we also denote the norm of a linear operator acting in $Y, L(Y)$ is a space of linear bounded operators acting in $Y$.

Let $\mathbf{l}_{\infty}(Y)$ be a space of sequences $\alpha=\left\{\alpha_{i}\right\}_{i=1}^{\infty}, \alpha_{i} \in Y, i=1,2, \ldots$, such that

$$
\sup _{i \geq 1}\left\|\alpha_{i}\right\|<\infty
$$

with the norm

$$
\|\alpha\|_{\mathbf{l}_{\infty}(Y)}=\sup _{i \geq 1}\left\|\alpha_{i}\right\|
$$

Definition. A function $x: \mathbf{R} \rightarrow Y$ absolutely continuous in each $\left[\tau_{i}, \tau_{i+1}\right)$ is a solution of the impulsive equation (1),(5), if for $t \neq \tau_{i}$ it satisfies (1) and for $t=\tau_{i}$ it satisfies (5).

The solution is assumed to be right continuous.

Further we need the following hypotheses.

(H1) $B_{i}: Y \rightarrow Y, i=1,2, \ldots$ are linear bounded operators , $A(t)$ is a continuous operator function with values in $L(Y), f(t)$ is a continuous function with values in $Y$. 
(H2) $B_{i}: Y \rightarrow Y$ have bounded inverse operators.

(H3) There exists $\sigma>0$ such that $\tau_{i+1}-\tau_{i}<\sigma, i=1,2, \ldots$.

(H4) There exists $\rho>0$ such that $\tau_{i+1}-\tau_{i}>\rho, i=1,2, \ldots$.

Suppose (H1) and (H2) hold. Then the impulsive equation (1),(5), $x(0)=$ $\alpha$ has one and only one solution that can be presented as (Bainov, Kostadinov and Myshkis, 1988)

$$
x(t)=X(t) \alpha+\int_{0}^{t} C(t, s) f(s) d s+\sum_{0<\tau_{i} \leq t} C\left(t, \tau_{i}\right) \alpha_{i},
$$

where the evolution operator

$$
C(t, s)=X(t) X^{-1}(s) .
$$

Here $X(t)$ is the solution of the operator equation

$$
\begin{gathered}
\dot{X}(t)+A(t) X(t)=0, \\
X\left(\tau_{i}\right)=B_{i} X\left(\tau_{i}-0\right), i=1,2, \ldots, \\
X(0)=I,
\end{gathered}
$$

where $I: Y \rightarrow Y$ is the identity operator.

Obviously $C(t, s)$ satisfies the semi-group equality

$$
C(t, s)=C(t, \tau) C(\tau, s) \text {. }
$$

Theorem 2.1 Suppose (H1),(H2) and (H3) hold and the solution of the problem (4),(5), $x(0)=0$ is bounded for any $\alpha=\left\{\alpha_{i}\right\}_{i=1}^{\infty} \in \mathbf{l}_{\infty}(Y)$.

Then there exist positive constants $N$ and $\nu$ such that the inequality

$$
\|X(t)\| \leq N \exp (-\nu t)
$$

holds.

Proof. By (7) the solution of the initial problem $(4),(5), x(0)=0$ has the representation

$$
x(t)=\sum_{0<\tau_{i} \leq t} C\left(t, \tau_{i}\right) \alpha_{i}
$$


Thus for any $t$ the right-hand side of (11) is a bounded linear operator acting from $\mathbf{l}_{\infty}(Y)$ to $Y$ since

$$
\|x(t)\| \leq \sum_{0<\tau_{i} \leq t}\left\|C\left(t, \tau_{i}\right)\right\| \cdot\|\alpha\|_{\mathbf{1}_{\infty}(Y)},
$$

where $\alpha=\left(\alpha_{1}, \alpha_{2}, \ldots, \alpha_{i}, \ldots\right) \in \mathbf{l}_{\infty}(Y)$. By the hypothesis of the theorem for any sequence $\alpha \in \mathbf{l}_{\infty}(Y)$ the solution $x(t)$ is bounded. Therefore the uniform boundedness principle implies that there exists $k>0$ such that

$$
\|x(t)\| \leq k\|\alpha\|_{\mathbf{l}_{\infty}} \text { for any } \alpha \in \mathbf{l}_{\infty} .
$$

Substituting of (8) in (11) gives

$$
\left\|\sum_{0<\tau_{i} \leq t} X(t) X^{-1}\left(\tau_{i}\right) \alpha_{i}\right\| \leq k\|\alpha\|_{\mathbf{l}_{\infty}} .
$$

Setting $\alpha_{1}=X\left(\tau_{1}\right) y,\|y\|=1$ for $\tau_{1} \leq t<\tau_{2}$ we obtain

$$
\|X(t)\| \leq k\left\|X\left(\tau_{1}\right)\right\|
$$

and $k \geq 1$.

By setting

$$
\alpha_{1}=\frac{X\left(\tau_{1}\right) y}{\left\|X\left(\tau_{1}\right)\right\|}, \alpha_{2}=\frac{X\left(\tau_{2}\right) y}{\left\|X\left(\tau_{2}\right)\right\|},\|y\|=1,
$$

we obtain from (12) for $t=\tau_{2}$

$$
\left\|X\left(\tau_{2}\right) y\right\|\left[\left(\left\|X\left(\tau_{1}\right)\right\|\right)^{-1}+\left(\left\|X\left(\tau_{2}\right)\right\|\right)^{-1}\right] \leq k .
$$

As $y$ is an arbitrary vector in $\mathbf{R}^{n}$ such that $\|y\|=1$ then

$$
\left\|X\left(\tau_{2}\right)\right\|\left[\left(\left\|X\left(\tau_{1}\right)\right\|\right)^{-1}+\left(\left\|X\left(\tau_{2}\right)\right\|\right)^{-1}\right] \leq k,
$$

therefore

$$
1+\frac{\left\|X\left(\tau_{2}\right)\right\|}{\left\|X\left(\tau_{1}\right)\right\|} \leq k
$$

Hence

$$
\left\|X\left(\tau_{2}\right)\right\| \leq(k-1)\left\|X\left(\tau_{1}\right)\right\| .
$$


Without loss of generality we can assume $k>1$.

By setting

$$
\alpha_{1}=\frac{X\left(\tau_{1}\right) y}{\left\|X\left(\tau_{1}\right)\right\|}, \alpha_{2}=\frac{X\left(\tau_{2}\right) y}{\left\|X\left(\tau_{2}\right)\right\|}, \alpha_{3}=\frac{X\left(\tau_{3}\right) y}{\left\|X\left(\tau_{3}\right)\right\|},\|y\|=1,
$$

we obtain from (12) for $t=\tau_{3}$

$$
\left\|X\left(\tau_{3}\right) y\right\|\left[\left(\left\|X\left(\tau_{1}\right)\right\|\right)^{-1}+\left(\left\|X\left(\tau_{2}\right)\right\|\right)^{-1}+\left(\left\|X\left(\tau_{3}\right)\right\|\right)^{-1}\right] \leq k .
$$

As $y \in \mathbf{R}^{n}$ is arbitrary such that $\|y\|=1$ then (13) gives

$$
1+\frac{\left\|X\left(\tau_{3}\right)\right\|}{\left\|X\left(\tau_{1}\right)\right\|(k-1)}+\frac{\left\|X\left(\tau_{3}\right)\right\|}{\left\|X\left(\tau_{1}\right)\right\|} \leq k .
$$

Thus

$$
\left\|X\left(\tau_{3}\right)\right\| \leq\left\|X\left(\tau_{1}\right)\right\|(k-1)^{2} / k .
$$

Now we prove by induction that

$$
\left\|X\left(\tau_{j+1}\right)\right\| \leq\left\|X\left(\tau_{1}\right)\right\|(k-1)^{j} / k^{j-1}
$$

for any positive integer $j$.

Suppose that $\left\|X\left(\tau_{i+1}\right)\right\| \leq\left\|X\left(\tau_{1}\right)\right\|(k-1)^{i} / k^{i-1}, i \leq j$.

Then by setting in (12)

$$
\alpha_{1}=\frac{X\left(\tau_{1}\right) y}{\left\|X\left(\tau_{1}\right)\right\|}, \ldots, \alpha_{j+1}=\frac{X\left(\tau_{j+1}\right) y}{\left\|X\left(\tau_{j+1}\right)\right\|}, \alpha_{j+2}=\frac{X\left(\tau_{j+2}\right) y}{\left\|X\left(\tau_{j+2}\right)\right\|},
$$

$\|y\|=1$, we obtain for $t=\tau_{j+2}$

$$
1+\frac{\left\|X\left(\tau_{j+2}\right)\right\|}{\left\|X\left(\tau_{1}\right)\right\|}\left[1+\frac{1}{k-1}+\frac{k}{(k-1)^{2}}+\ldots+\frac{k^{j-1}}{(k-1)^{j}}\right] \leq k .
$$

As a sum of geometric progression

$$
\sum_{i=0}^{j-1} \frac{k^{i}}{(k-1)^{i+1}}=\frac{\frac{k^{j}}{(k-1)^{j+1}}-\frac{1}{k-1}}{\frac{k}{k-1}-1}=\frac{k^{j}}{(k-1)^{j}}-1,
$$

then

$$
\frac{\left\|X\left(\tau_{j+2}\right)\right\|}{\left\|X\left(\tau_{1}\right)\right\|}\left[1+\frac{k^{j}}{(k-1)^{j}}-1\right] \leq k-1
$$


Hence $\left\|X\left(\tau_{j+2}\right)\right\| \leq\left\|X\left(\tau_{1}\right)\right\|(k-1)^{j+1} / k^{j}$.

Let in $(12)$

$$
\alpha_{1}=\alpha_{2}=\ldots=\alpha_{j}=0, \alpha_{j+1}=X\left(\tau_{j+1}\right) y,\|y\|=1 .
$$

Then

$$
\|X(t)\| \leq k\left\|X\left(\tau_{j+1}\right)\right\| \leq\left\|X\left(\tau_{1}\right)\right\|(k-1)^{j} / k^{j-2}
$$

for $\tau_{j+1} \leq t<\tau_{j+2}$. Since by the hypothesis of the theorem $t<(j+2) \sigma$, i.e. $j>t / \sigma-2$, then

$$
\ln (\|X(t)\|) \leq \ln \left(\left\|X\left(\tau_{1}\right)\right\|\right)-(t / \sigma-2) \ln [k /(k-1)]+2 \ln k .
$$

By assuming

$$
\begin{gathered}
\nu=\ln [k /(k-1)] / \sigma \\
N_{1}=\left\|X\left(\tau_{1}\right)\right\| k^{4} /(k-1)^{2}, \\
N=\max \left\{N_{1}, \sup _{0 \leq t<\tau_{1}}[\exp (\nu t)\|X(t)\|]\right\}
\end{gathered}
$$

we obtain the inequality (10). The proof of the theorem is complete.

Definition. The equation (1),(2) is said to be exponentially stable if there exist positive constants $N$ and $\nu$ such that for any solution of the equation $(4),(2)$ the inequality

$$
\|x(t)\| \leq N \exp (-\nu t)\|x(0)\|
$$

holds.

Corollary . Suppose the hypotheses of Theorem 2.1 hold. Then the equation (1), (2) is exponentially stable.

Consider an additional hypothesis.

(H5) There exist positive constants $b$ and $M$ such that

$$
\sup _{i}\left\|B_{i}\right\| \leq b, \quad \int_{\tau_{i}}^{\tau_{i+1}}\|A(s)\| d s \leq M, i=1,2, \ldots .
$$


Theorem 2.2 Suppose the hypotheses (H1)-(H3) and (H5) hold and the solution of the problem (4),(5), $x(0)=0$ is bounded for any $\alpha=\left\{\alpha_{i}\right\}_{i=1}^{\infty} \in$ $\mathbf{l}_{\infty}(Y)$.

Then there exist positive constants $N$ and $\nu$ such that

$$
\|C(t, s)\| \leq N \exp [-\nu(t-s)]
$$

$0 \leq s<t<\infty$

Proof. We fix a positive integer $p$. Then for any $u \in Y \quad C\left(t, \tau_{p}\right) u$ is the solution of the problem (4),(5), $x(0)=0, \alpha_{i}=0, i \neq p, \alpha_{p}=u$.

By repeating the proof of Theorem 2.1 we obtain

$$
\left\|C\left(t, \tau_{p}\right)\right\| \leq N_{p} \exp \left[-\nu\left(t-\tau_{p}\right)\right]
$$

with

$$
N_{p}=\max \left\{\left\|C\left(\tau_{p+1}, \tau_{p}\right)\right\| k^{4} /(k-1)^{2}, \sup _{\tau_{p} \leq t<\tau_{p+1}}\left[\exp \left(\nu\left(t-\tau_{p}\right)\right)\left\|C\left(t, \tau_{p}\right)\right\|\right]\right\} .
$$

Now we have to show that $N_{p}$ can be chosen independently of $p$.

The operator $C\left(t, \tau_{p}\right)$ is the solution of the operator equation

$$
\dot{C}\left(t, \tau_{p}\right)+A(t) C\left(t, \tau_{p}\right)=0, t \in\left[\tau_{p}, \tau_{p+1}\right),
$$

where $C\left(\tau_{p}, \tau_{p}\right)=I, I: Y \rightarrow Y$ is the identity operator,

$$
C\left(\tau_{p+1}, \tau_{p}\right)=B_{p+1} C\left(\tau_{p+1}-0, \tau_{p}\right) .
$$

Thus for $t \in\left[\tau_{p}, \tau_{p+1}\right)$ the operator $C\left(t, \tau_{p}\right)$ is the solution of the integral equation

$$
C\left(t, \tau_{p}\right)=I-\int_{\tau_{p}}^{t} A(\xi) C\left(\xi, \tau_{p}\right) d \xi
$$

By the Gronwall-Bellman inequality we obtain

$$
\left\|C\left(t, \tau_{p}\right)\right\| \leq \exp \left\{\int_{\tau_{p}}^{t}\|A(\xi)\| d \xi\right\} .
$$

Therefore (14) gives

$$
\left\|C\left(t, \tau_{p}\right)\right\| \leq e^{M}, \quad t \in\left(\tau_{p}, \tau_{p+1}\right) .
$$


For $t=\tau_{p+1}$

$$
\left\|C\left(\tau_{p+1}, \tau_{p}\right)\right\| \leq\left\|B_{p+1}\right\|\left\|C\left(\tau_{p+1}-0, \tau_{p}\right)\right\| \leq b e^{M} .
$$

Hence

$$
\left\|C\left(t, \tau_{p}\right)\right\| \leq N_{2} \exp \left[-\nu\left(t-\tau_{p}\right)\right]
$$

where

$$
N_{2}=\max \left\{b e^{M} k^{4} /(k-1)^{2}, b e^{\nu \sigma+M}, e^{\nu \sigma+M}\right\} .
$$

Now let $s \in[0, \infty)$ be arbitrary. Suppose $\tau_{p}$ is the least of all $\tau_{p} \geq s$. Then

$$
\begin{gathered}
\|C(t, s)\|=\left\|C\left(t, \tau_{p}\right) C\left(\tau_{p}, s\right)\right\| \leq\left\|C\left(t, \tau_{p}\right)\right\|\left\|C\left(\tau_{p}, s\right)\right\| \leq \\
\leq N_{2} \exp \left[-\nu\left(t-\tau_{p}\right)\right] \cdot N_{2} \exp \left[-\nu\left(\tau_{p}-s\right)\right]=N_{2}^{2} \exp [-\nu(t-s)]
\end{gathered}
$$

The latter inequality may be proven similar to the proof of inequalities (16), (17).

Therefore $\|C(t, s)\| \leq N \exp [-\nu(t-s)]$, with $N=N_{2}^{2}$.

The proof of the theorem is complete.

\section{Main results}

Main results of this work are the following.

Theorem 3.1 Suppose the hypotheses (H1)-(H5) hold and the solution of (4),(5), $x(0)=0$ is bounded on the half-line for any $\alpha=\left\{\alpha_{i}\right\}_{i=1}^{\infty} \in \mathbf{l}_{\infty}(Y)$.

Then each solution $x(t)$ of the problem (1),(5) with $f, \alpha$ satisfying (3),(6), is bounded for $t \geq 0$.

Proof. By Theorems 2.1, 2.2 there exist positive constants $N$ and $\nu$ such that

$$
\begin{gathered}
\|C(t, s)\| \leq N \exp [-\nu(t-s)], \\
\|X(t)\| \leq N \exp (-\nu t) .
\end{gathered}
$$

The solution $x$ has the representation (7). Thus

$$
\|x(t)\| \leq\|X(t)\|\|x(0)\|+\sum_{0<\tau_{i} \leq t}\left\|C\left(t, \tau_{i}\right)\right\|\left\|\alpha_{i}\right\|+
$$




$$
\begin{gathered}
+\int_{0}^{t}\|C(t, s)\|\|f(s)\| d s \leq N \exp (-\nu t)\|x(0)\|+ \\
+\sum_{i=1}^{\infty} N \exp \left[-\nu\left(t-\tau_{i}\right)\right]\left\|\alpha_{i}\right\|+\int_{0}^{t} N \exp [-\nu(t-s)]\|f(s)\| d s .
\end{gathered}
$$

Consider the second term. Let $\tau_{k}$ be the greatest of all $\tau_{i}<t$. Then

$$
t-\tau_{k-1} \geq \tau_{k}-\tau_{k-1}>\rho, t-\tau_{k-2}>2 \rho, \ldots, t-\tau_{1}>(k-1) \rho .
$$

Therefore

$$
\sum_{j=1}^{\infty} \exp \left[-\nu\left(t-\tau_{j}\right)\right] \leq \sum_{j=0}^{\infty} \exp (-\nu j \rho)=\frac{1}{1-\exp (-\nu \rho)}
$$

Hence

$$
\|x(t)\| \leq N\|x(0)\|+\frac{N\|\alpha\|_{\mathbf{1}_{\infty}(X)}}{1-\exp (-\nu \rho)}+\frac{N}{\nu} \sup _{s \geq 0}\|f(s)\| .
$$

Thus $x(t)$ is bounded for $t \geq 0$, which completes the proof of the theorem.

Theorem 3.2 Suppose the hypotheses of Theorem 3.1 hold.

(a) Then

$$
\lim _{n \rightarrow \infty}\left\|\alpha_{n}\right\|=0, \lim _{t \rightarrow \infty}\|f(t)\|=0
$$

imply that for any solution $x$ of (1),(5)

$$
\lim _{t \rightarrow \infty}\|x(t)\|=0 \text {. }
$$

(b) Suppose that there exist positive constants $N_{1}$ and $\lambda$ such that

$$
\left\|\alpha_{n}\right\| \leq N_{1} \exp (-\lambda n), \quad\|f(t)\| \leq N_{1} \exp (-\lambda t)
$$

Then for any solution $x$ of (1),(5) there exist positive constants $N_{0}$ and $\nu_{0}$ such that

$$
\|x(t)\| \leq N_{0} \exp \left(-\nu_{0} t\right)
$$


Proof. (a) By Theorems 2.1, 2.2 as in the proof of Theorem 3.1 one obtains

$$
\begin{gathered}
\|x(t)\| \leq N \exp (-\nu t)\|x(0)\|+\sum_{0<\tau_{i} \leq t} N \exp \left[-\nu\left(t-\tau_{i}\right)\right]\left\|\alpha_{i}\right\|+ \\
+\int_{0}^{t} N \exp [-\nu(t-s)]\|f(s)\| d s
\end{gathered}
$$

We fix $\varepsilon>0$. Let $t_{1}>0$ be such that

$$
t_{1}>-\frac{1}{\nu} \ln \left\{\frac{\varepsilon}{3 N\|x(0)\|}\right\} .
$$

Then the first term in the sum

$$
N \exp (-\nu t)\|x(0)\|<\varepsilon / 3 .
$$

Let $\tilde{t}$ be such that for $\tau_{i} \geq \tilde{t}$

$$
\left\|\alpha_{i}\right\|<\frac{\varepsilon}{6 N[1-\exp (-\nu \rho)]}
$$

and $t_{2}>\tilde{t}$ be such that for $t>t_{2}$

$$
\sum_{0<\tau_{i} \leq \tilde{t}} N \exp \left[-\nu\left(t-\tau_{i}\right)\right]\left\|\alpha_{i}\right\|<\frac{\varepsilon}{6} .
$$

Then similar to the proof of Theorem 3.1 we evaluate the second term for $t>t_{2}$

$$
\begin{aligned}
& \sum_{0<\tau_{i} \leq t} N \exp \left[-\nu\left(t-\tau_{i}\right)\right]\left\|\alpha_{i}\right\|=\sum_{0<\tau_{i} \leq \tilde{t}} N \exp \left[-\nu\left(t-\tau_{i}\right)\right]\left\|\alpha_{i}\right\|+ \\
&+\sum_{\tilde{t}<\tau_{i} \leq t} N \exp \left[-\nu\left(t-\tau_{i}\right)\right]\left\|\alpha_{i}\right\| \leq \frac{\varepsilon}{6}+\sum_{j=0}^{\infty} N \exp (-\nu j \rho) \frac{\varepsilon}{6 N[1-\exp (-\nu \rho)]}< \\
&<\frac{\varepsilon}{6}+\frac{\varepsilon}{6}=\frac{\varepsilon}{3} .
\end{aligned}
$$

The corresponding estimation of the third term

$$
\int_{0}^{t} N \exp [-\nu(t-s)]\|f(s)\| d s \quad \text { for } \quad \lim _{s \rightarrow \infty}\|f(s)\|=0
$$


is given in (Massera and Schäffer, 1966). Thus for a certain $t_{3}$

$$
\int_{0}^{t} N \exp [-\nu(t-s)]\|f(s)\| d s<\frac{\varepsilon}{3},
$$

for each $t>t_{3}$.

Therefore for $t>\max \left\{t_{1}, t_{2}, t_{3}\right\}$

$$
\|x(t)\|<\frac{\varepsilon}{3}+\frac{\varepsilon}{3}+\frac{\varepsilon}{3}=\varepsilon .
$$

Since $\varepsilon>0$ is arbitrary we obtain $\lim _{t \rightarrow \infty}\|x(t)\|=0$.

(b) For obtaining an exponential estimate of $x(t)$ we have to evaluate each of three above terms.

The first term

$$
N \exp (-\nu t)\|x(0)\|
$$

obviously has the exponential estimate, the third one under the hypotheses of the corollary also can be estimated as (Massera and Schäffer, 1966)

$$
\int_{0}^{t} N \exp [-\nu(t-s)]\|f(s)\| d s \leq N_{3} \exp \left(-\nu_{3} t\right),
$$

with certain positive constants $N_{3}$ and $\nu_{3}$.

Now we prove that there exist positive constants $N_{2}$ and $\nu_{2}$ such that

$$
\sum_{0<\tau_{i} \leq t} N \exp \left[-\nu\left(t-\tau_{i}\right)\right]\left\|\alpha_{i}\right\|<N_{2} \exp \left(-\nu_{2} t\right) .
$$

Let $\tau_{k} \leq t<\tau_{k+1}$. Then denoting

$$
\beta=\sum_{0<\tau_{i} \leq t} N \exp \left[-\nu\left(t-\tau_{i}\right)\right]\left\|\alpha_{i}\right\|
$$

we obtain

$$
\beta \leq \sum_{i=1}^{k} N \exp \left[-\nu\left(t-\tau_{i}\right)\right] N_{1} \exp (-\lambda i) .
$$

Since $t-\tau_{i} \geq(k-i) \rho($ see the proof of Theorem 3.1) then

$$
\beta \leq N N_{1} \sum_{i=1}^{k} \exp [-\nu(k-i) \rho] \exp (-\lambda i)=
$$




$$
\begin{gathered}
=N N_{1} \exp (-\nu \rho k) \sum_{i=1}^{k} \exp [(\nu \rho-\lambda) i] \leq \\
\leq N N_{1} \exp (-\nu \rho k) \frac{\exp [(\nu \rho-\lambda)(k+1)]}{\exp (|\nu \rho-\lambda|)-1}= \\
=\frac{N N_{1} \exp (\nu \rho)}{\exp (|\nu \rho-\lambda|)-1} \exp [-\lambda(k+1)] .
\end{gathered}
$$

The hypothesis (H3) gives that $t<\tau_{k+1}<(k+1) \sigma$. Therefore

$$
\beta \leq \frac{N N_{1} \exp (\nu \rho)}{\exp (|\nu \rho-\lambda|)-1} \exp \left(-\frac{\lambda}{\sigma} t\right)=N_{2} \exp \left(-\nu_{2} t\right)
$$

with $\nu_{2}=-\lambda / \sigma$

$$
N_{2}=\frac{N N_{1} \exp (\nu \rho)}{\exp (|\nu \rho-\lambda|)-1} .
$$

Denoting

$$
\begin{gathered}
N_{0}=\max \left\{N, N_{2}, N_{3}\right\}, \\
\nu_{0}=\min \left\{\nu, \nu_{2}, \nu_{3}\right\}
\end{gathered}
$$

we obtain

$$
\|x(t)\| \leq N_{0} \exp \left(-\nu_{0} t\right)
$$

which completes the proof.

Corollary 3.3. Suppose $Y=\mathbf{R}$ (scalar case), (H1)-(H5) hold and the solution of (4),

$$
x\left(\tau_{i}\right)=B_{i} x\left(\tau_{i}-0\right)+\operatorname{sign}\left[B_{i} X\left(\tau_{i}-0\right)\right], i=1,2, \ldots,
$$

$x(0)=0$ is bounded on the half-line. Here $X$ is the function from the representation (7), sign $u=u /|u|$.

Then each solution of $(1),(5)$ is bounded on $[0, \infty)$ for any $f, \alpha$ satisfying (3) and (6) correspondingly.

Proof. By the hypothesis of the corollary there exists $Q>0$ such that $|x(t)| \leq Q, t \geq 0$, where $x$ is the solution of (4),(18), $x(0)=0$. 
As $\alpha_{i}=X\left(\tau_{i}\right) /\left|X\left(\tau_{i}\right)\right|$, then (7) implies

$$
|x(t)|=\left|\sum_{0<\tau_{i} \leq t} C\left(t, \tau_{i}\right) X\left(\tau_{i}\right) /\right| X\left(\tau_{i}\right)|\quad| \leq Q .
$$

However

$$
C\left(t, \tau_{i}\right) X\left(\tau_{i}\right)
$$

have the same sign for any $i=1,2, \ldots, \tau_{i} \leq t$, for a fixed $t$. Therefore

$$
\left|\sum_{0<\tau_{i} \leq t} C\left(t, \tau_{i}\right) X\left(\tau_{i}\right) /\right| X\left(\tau_{i}\right)||=\sum_{0<\tau_{i} \leq t}\left|C\left(t, \tau_{i}\right)\right| \leq Q .
$$

Hence for any $\alpha=\left\{\alpha_{i}\right\}_{i=1}^{\infty} \in \mathbf{l}_{\infty}$ the solution $x$ of $(4),(5)$ is bounded :

$$
|x(t)|=\left|\sum_{0<\tau_{i} \leq t} C\left(t, \tau_{i}\right) \alpha_{i}\right| \leq \sum_{0<\tau_{i} \leq t}\left|C\left(t, \tau_{i}\right)\right| \cdot\|\alpha\|_{\mathbf{l}_{\infty} \leq Q\|\alpha\|_{\mathbf{l}_{\infty}} .} .
$$

Thus the hypotheses of Theorem 3.1 are satisfied. Therefore each solution of $(1),(5)$ is bounded on the half-line whenever $f, \alpha$ satisfy $(3),(6)$ correspondingly.

One can apply Theorems 2,1, 2.2 to ordinary differential equations without impulses.

Corollary 3.4. Suppose there exists a positive constant $\eta$ such that for any sequence $\alpha=\left\{\alpha_{i}\right\}_{i=1}^{\infty} \in \mathbf{l}_{\infty}(Y)$ the equation

$$
\dot{x}(t)+A(t) x(t)=\sum_{i=1}^{\infty} \alpha_{i} \delta(t-\eta i)
$$

has a bounded solution.

Then there exist positive constants $N$ and $\nu$ such that for the solution of (4) the estimates (10) and (15) are valid.

Here $\delta(t-\eta i)$ is a delta function, the derivative in the left-hand side and the equality are understood in the distributional sense. 


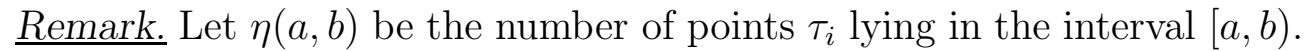
It is to be noted that the usual condition (Bainov et al., 1989)

$$
\lim _{\omega \rightarrow \infty} \frac{\eta(t, t+\omega)}{\omega}=q<\infty
$$

is more restrictive than both hypotheses (H3) and (H4).

\section{Exponential estimates of impulsive and con- tinuous solutions}

Since many results on exponential estimation of the differential equation (1) are known the following question is of special interest. Let any solution of the problem $(1), x(0)=0$ be bounded on $[0, \infty)$ for any bounded on $[0, \infty)$ right-hand side $f$. Does this property preserve for the impulsive problem $(1),(2)$ ? Boundedness of solutions is connected with exponential estimates of an evolution operator. The following examples illustrate that an exponential estimate for the impulsive equation does not imply an exponential estimate for the corresponding differential equation (1) and vice versa.

Example 1. The solution of the problem

$$
\dot{x}=0, x(i)=0.5 x(i-0), i=1,2, \ldots, x(0)=1
$$

can be estimated

$$
x(t) \leq 2 \exp (-t \ln 2)
$$

while the solution of

$$
\dot{x}=0, x(0)=1
$$

is constant.

Example 2. The equation $\dot{x}+x=f$ is exponentially stable. However for the impulsive equation

$$
\dot{x}+x=0, x(i)=e x(i-0), i=1,2, \ldots,
$$

we obtain $x(i)=x(0)$.

It is well known that the operator $X(t)$ in the representation (7) for the solution of the impulsive equation $(1),(5)$ can be defined if we know the 
corresponding operator $U(t)$ of the nonimpulsive equation (1) (Bainov et al., 1989)

$$
X(t)=U(t) U^{-1}\left(\tau_{i}\right) \prod_{j=1}^{i} B_{i-j+1} X\left(\tau_{i-j+1}\right) X^{-1}\left(\tau_{i-j}\right)
$$

for $t \in\left[\tau_{i}, \tau_{i+1}\right)$. If we know the evolution operator $G(t, s)=U(t) U^{-1}(s)$ of the non-impulsive equation, then the evolution operator of the impulsive equation (Bainov et al., 1989)

$$
C(t, s)=\left\{\begin{array}{l}
G(t, s), \tau_{i} \leq t, s<\tau_{i+1} \\
G\left(t, \tau_{i}\right)\left[\prod_{j=i}^{k+1} B_{j} G\left(\tau_{j}, \tau_{j-1}\right)\right] B_{k} G\left(\tau_{k}, s\right) \\
\tau_{k-1} \leq s<\tau_{k}<\tau_{i} \leq t<t_{i+1} \\
G\left(t, \tau_{i}\right)\left[\prod_{j=i}^{k-1} B_{j}^{-1} G\left(\tau_{j}, \tau_{j+1}\right)\right] B_{k}^{-1} G\left(\tau_{k}, s\right) \\
\tau_{i-1} \leq t<\tau_{i}<\tau_{k} \leq s<t_{k+1} .
\end{array}\right.
$$

Theorem 4.1 Let $X=\mathbf{R}$ (scalar case). Suppose there exists a positive constant $\varepsilon$ such that

$$
\left|\prod_{k=i}^{j} B_{k}\right| \geq \varepsilon
$$

for each $i, j, i, j=1,2, \ldots$, (H1)-(H3) hold and any solution of the initial problem (4),(5), $x(0)=0$ is bounded on $[0, \infty)$ for any bounded sequence $\left\{\alpha_{i}\right\}_{i=1}^{\infty}$.

Then there exist positive constants $N$ and $\nu$ such that the solution $U(t)$ of non-impulsive equation (4), $U(0)=1$, satisfies (10).

If, in addition, (H5) holds, then for $G(t, s)$ the estimate (15) is valid for certain $N, \nu>0$.

Proof. By Theorem 2.1 the hypotheses of the theorem imply that there exist $N_{1}>0, \nu>0$ such that

$$
|X(t)| \leq N_{1} \exp (-\nu t)
$$


where $X(t)$ is the solution of $(4),(2), \mathrm{x}(0)=1$. In the scalar case (19) can be rewritten as

$$
X(t)=U(t) \prod_{0<\tau_{i} \leq t} B_{i}
$$

Therefore

$$
|U(t)| \leq \frac{|X(t)|}{\varepsilon} \leq \frac{N_{1}}{\varepsilon} \exp (-\nu t) \leq N \exp (-\nu t),
$$

with $N=N_{1} / \varepsilon$.

Similarly (20) implies the estimate (15) for $G(t, s)$.

Theorem 4.2 Let the operators $B_{i}$ and $G(t, s)$ be commuting for each $t, s \geq 0, i=$ $1,2, \ldots$ Suppose any solution of the initial problem (1), $x(0)=0$ is bounded on $[0, \infty)$ for any bounded on $[0, \infty)$ right-hand side $f$ and there exists $Q>0$ such that

$$
\prod_{k=i}^{j}\left\|B_{k}\right\| \leq Q
$$

for each $i, j=1,2, \ldots$.

Then there exist positive constants $N$ and $\nu$ such that the solution $X$ of the problem (4),(2), $X(0)=I$, has an estimate (10).

If, in addition, there exists $M>0$ such that

$$
\int_{i}^{i+1}\|A(s)\| d s<M, i=1,2, \ldots
$$

then for $C(t, s)=X(t) X^{-1}(s)$ the estimate (15) is valid, with certain $N, \nu>0$.

Proof. The hypotheses of the theorem imply (Daleckii and Krein, 1974, p.127) that there exist $N_{1}, \nu>0$ such that

$$
\|U(t)\| \leq N_{1} \exp (-\nu t)
$$

Since $B_{i}$ and $U(t)=G(t, 0)$ are commuting, we obtain (21). Hence

$$
\|X(t)\| \leq\|U(t)\| \prod_{0<\tau_{i} \leq t}\left\|B_{i}\right\| \leq Q N_{1} \exp (-\nu t)=N \exp (-\nu t),
$$


with $N=N_{1} Q$.

Similarly by applying (20) one obtains (15), which completes the proof of the theorem.

\section{Acknowledgement}

This work was supported by Israel Ministry of Science and Technology and by the Centre for Absorption in Science, Ministry of Immigrant Absorption State of Israel.

\section{References}

Anokhin, A., Berezansky, L., and Braverman, E. Exponential stability of linear delay impulsive differential equations . Preprint funct-an/9311004.

Bainov, D. D., Kostadinov, S. I., and Myshkis, A. D. (1988). Differential and Integral Equations, 1 (2) , 233.

Bainov, D.D., Kostadinov, S.I, Nguyen Van Minh, and Zabreiko, P.P. (1993). International Journal of Theoretical Physics, 32, 1275.

Bainov, D. D., Kostadinov, S. I., and Zabreiko, P. P. (1989). International Journal of Theoretical Physics, 28, 797.

Bainov, D. D., Zabreiko, P. P., and Kostadinov, S. I. (1988). International Journal of Theoretical Physics, 27, 373.

Bressan, A., and Rampazzo, F. (1991). Journal of Optimization Theory and Applications, $\mathbf{7 1}$ (1), 67.

Daleckï, Ju. L., and Kreín, M. G. (1974). Stability of Solutions of Differential Equations in Banach Spaces, AMS, Providence, Rhode Island.

Massera, J. L., and Schäffer, J. (1966). Linear Differential Equations and Function Spaces, Academic Press, New York.

Zabreiko, P. P., Bainov, D. D., and Kostadinov, S. I. (1988). International Journal of Theoretical Physics, 27, 731. 